International Journal on Cybernetics \& Informatics (IJCI) Vol. 5, No. 4, August 2016

\title{
IMPEDANCE CARDIOGRAPHY FILTERING USING NON-NEGATIVE LEAST-MEAN-SQUARE ALGORITHM
}

\author{
Dr. K.ChandraBhushana Rao ${ }^{1}$ and Ms. Madhavi Mallam ${ }^{2}$ \\ ${ }^{1}$ Associate Professor, Department of Electronics and Communication Engineering, \\ University College of Engineering, Vizianagaram, JNTUK A.P., 535002, India. \\ ${ }^{2}$ Research Scholar in Department of Electronics and Communication Engineering, \\ Jawaharlal Nehru Technological University, Kakinada, A.P., 533003, India.
}

\begin{abstract}
In general using several signal acquisition methods are applied to get cardio-impedance signal to analyse the cardiac output. The analysis completely based on frequency information obtained after applying frequency selection filters and frequency shaping filters. Here proposing a constructive approach involves a developed Non-Negative LMS (NNLMS) followed by filtering techniques to measure and overcome the limitations of commonly used approaches. The proposed technique performance is analysed by considering different types of noise environments like fundamental one white noise and also sum of sinusoidal noise. The simulation results are useful to measure the performance and accuracy under different noise environments also a comparative analysis is done with the proposed work with existing methods under different performance metrics by the help of quantitative analysis of algorithms. Simulation results are found to be satisfactory in the analysis of cardiac output.
\end{abstract}

\section{KEYWORDS}

NNLMS, Cardiac Output, excess mean-square error

\section{INTRODUCTION}

To acquire cardiac output many traditional approaches are available out of these methods the Impedance Cardio Graphy (ICG) is one acquisition technique. ICG acquisition method [3] involves a repetitive and non-invasive procedure on a beat-by beat basis. ICG is also cost effective and a very sensitive acquisition system of cardiac output. Out of many advantages of ICG methodology the major problem is it was very sensitive to the nose induced by shock or ventilation and movement of body while acquiring the cardiac signal. Based on the operation, the ICG signal $\mathrm{Z}=\mathrm{Z0}+\not \mathrm{Z}$ can be processed and analysed with its differential operator signal is processed directly in order to obtain fiducial points [3] like :Opening of aortic valves (B), aortic valve closure (X) and the maximum value of $\mathrm{dZ} / \mathrm{dt}$ following the opening of valves (C) [3] as shown in figure 1. So many researchers are working towards the analysis of these methods to DOI: 10.5121/ijci.2016.5413 
model a fine quantitative analysis and improvement in the performance analysis of cardiac output under different sensitive noise environments. In this work considered Non-Negativity is one of the limitations that can be applied on estimation of parameter [1] due to this physically unreasonable solutions can be avoid an can comply the characteristics of natural phenomena. This non negativity will also appear even in some processing schemes like audio processing, image processing, remote sensing and neuroscience [1]. The non-negativity constraints can be reduced by several processing schemes proposed namely NNLMS algorithms and its three different approaches like Normalisation, Exponential and Sign-Sign. Also transient behaviour and a recursive model analysis which is developed by weights behaviour of adaptive filters [1].

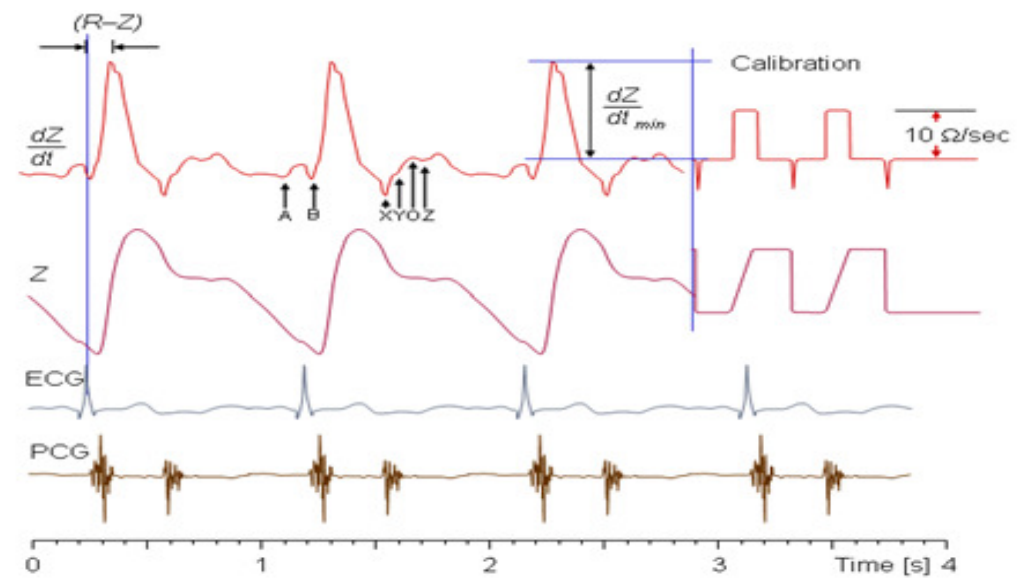

Figure 1: Sample ICG waveform taken from Ref [3].

\section{SELECTED METHOdology}

\subsection{An Artifact Canceller:}

Initially impedance sensor is used to collect thoracic impedance signal $\mathrm{z}(\mathrm{n})$ which consist of respiratory artifact and Reference signal $r(n)$ is obtained from the respiratory sensor[2]. The respiratory sensor to acquire reference signal there has some delay with respect to the respiratory artifact due to the movement of thorax cage [2]. To equalize a delay is introduce in the impedance signal $z(n)$. As illustrated in figure 2 an FIR filter and its weights are updated continuously to minimize the error e(n) with the help of adaptive algorithm namely Least Mean Square also name as statistical gradient search algorithm. Also the output of filter $\hat{r}^{\wedge}(\mathrm{n})$ is subtracted from delayed input impedance signal $\mathrm{z}(\mathrm{n})$ there after applied continuously error minimization scheme with algorithms. 
International Journal on Cybernetics \& Informatics (IJCI) Vol. 5, No. 4, August 2016

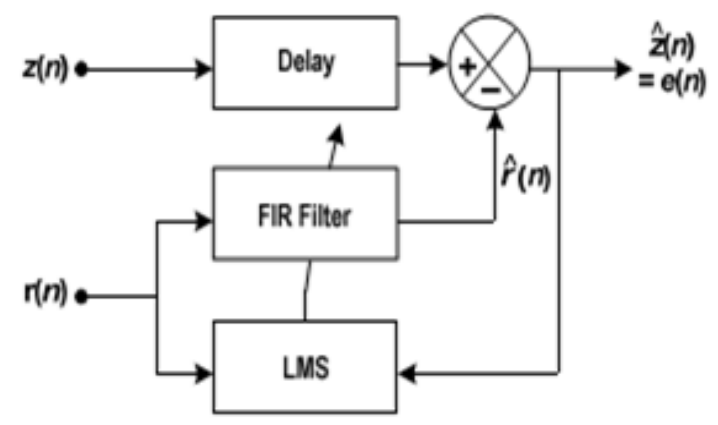

Figure 2: Artifact Canceller using Digital FIR

In this illustration the major purpose of algorithms is only to control or update the filter weights of Digital FIR filter in order to minimize the error.

\subsection{Impedance Signal Analysis}

The impedance sensor output is initially processed through a low pass filter as a part of eliminating high frequencies there by using 2-point differentiation we can obtain ICG waveform. Even after processing In ICG waveform there will be presence of respiratory artifact especially in the post-exercise recordings [2]. So in order to remove the artifact an artifact canceller illustrated in figure 2 was applied on impedance signal $z(n)$, by considering $\mu=0.0044$ for adaptation. The results are found to be satisfactory for tap length $M \approx 200$ and sample delay $\approx 70$. Because of large value of $d Z / d t$ is a useful parameter in the evaluation of stroke volume the same data is processed through the filter with specified inputs of filters. The percentage error was then calculated between the peaks of the standard waveforms and the filtered data with various cut-off frequencies [4].

The peak of $d Z / d t$ error values are illustrated with respect to different cut-off frequencies. The peak $d Z / d t$ error in all environments is less than 2 percent for a cut off frequency of less than 50 per cent of the heart rate [4].

Assume a linear model of system with inputs and outputs as illustrated in the following equation

$$
R(n)=\alpha^{* T} a(n)+z(n)
$$

The reference signal $R(n)$ and input signal $a(n)$ are assumed to be stationary and zero mean. The error also assumed to be stationary and minimum variance with zero mean. So based on physical constraints non negativity will be presented in the estimated coefficients to minimize that adaptive system is proposed with improved algorithms in order to minimize the Mean Squre Error with good number filter taps 


$$
\begin{aligned}
& \alpha^{o}=\underset{\alpha}{\arg \min } E\left\{\left[R(n)-\alpha^{T} a(n)\right]^{2}\right\} \\
& \text { subject to } \quad \alpha_{i} \geq 0, \forall i
\end{aligned}
$$

The non-negativity problem can be solve by updating the Digital FIR filter weights using a NNLMS algorithm with the following weight update equation

$$
\alpha(n+1)=\alpha(n)+\eta D_{\alpha}(n) e(n) a(n)
$$

The above weight update equation is further normalised in order to improve the performance of weight updating in process of minimisation of mean square error so the modified weight update recursive relation is given as

$$
\alpha_{N}(n+1)=\alpha_{N}(n)+\frac{\eta}{x^{T}(n) x(n)} D_{\alpha}(n) e N^{(n) x(n)}
$$

So all the above quatitative analysis are very usefull to remove artifacts in ICG waveform threre by the analysis of cardiac data is very effective in application aspects.

\section{SIMULATION RESULTS}

In the following illustration the collected ECG from impedence sensors are given in figure 3 shows that the recorded $z(n)$ also the processed data using the proposed methodology is displayed in figure 4 shows that Mean Square Error analysis under different environments. ICG waveform obtained after processed by differentiation of $z(n)$ is shown as first one. The impedance waveform after adaptive cancellation of the artifact is shown as second one in Figure 4. ICG obtained by differentiating this waveform shows almost no effect of respiration, making it easy to detect the $\mathrm{B}$ and X points and these are found to be consistent with simultaneously acquired PCG [2]. After processed the data through the adaptive filter with algorithms support the performance comparison is illustrated in following tabulation which yields the NNLMS will be the optimised algorithms in the removal of artifacts in ICG for better analysis of cardiac data also in the tabulation given the normalised NNLMS SNR evaluation to compare the existing methods in table 1 . 
International Journal on Cybernetics \& Informatics (IJCI) Vol. 5, No. 4, August 2016

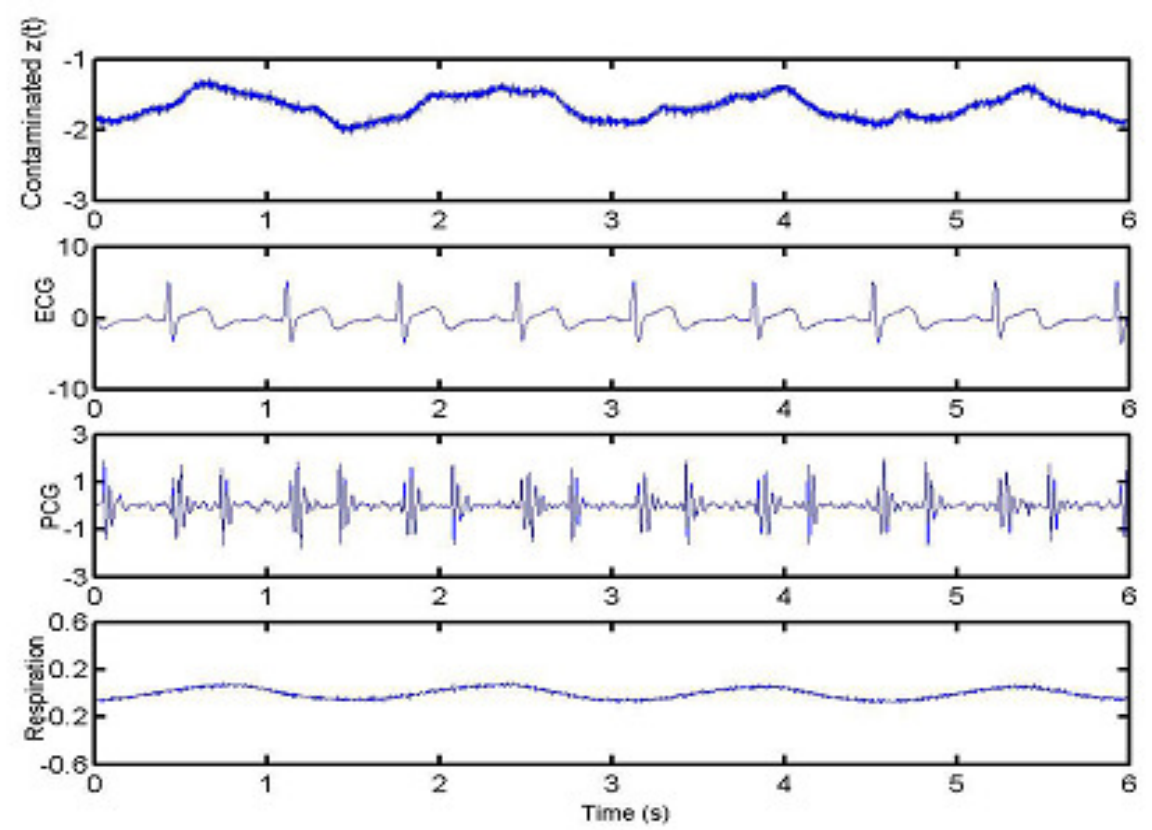

Figure 3. ICG, ECG,PCG in post exercise condition[2].

Table 1: SNR comparision with different algorithms in (a)ICG, (b)ECG, (c)PCG

\begin{tabular}{|l|l|l|l|l|}
\hline & SNR dB & (a) & (b) & (c) \\
\hline EA10 & -10 & -0.03 & 4.81 & 5.31 \\
\hline & -6 & 3.93 & 8.26 & 8.79 \\
\hline & -3 & 6.84 & 10.62 & 11.93 \\
\hline & 0 & 9.74 & 14.15 & 13.73 \\
\hline & 3 & 12.42 & 16.11 & 16.81 \\
\hline & 6 & 14.89 & 17.1 & 18.06 \\
\hline & 10 & 17,61 & 19.71 & 19.86 \\
\hline
\end{tabular}


International Journal on Cybernetics \& Informatics (IJCI) Vol. 5, No. 4, August 2016

\begin{tabular}{|l|l|l|l|l|}
\hline $\begin{array}{l}\text { NN } \\
\text { LMS-based }\end{array}$ & -10 & 12.43 & 2.63 & 1.23 \\
\hline & -6 & 16.5 & 3.32 & 4.17 \\
\hline & -3 & 18.97 & 6.18 & 6.92 \\
\hline & 0 & 21.88 & 8.67 & 9.71 \\
\hline & 3 & 24.51 & 11.12 & 12.47 \\
\hline & 6 & 26.62 & 13.65 & 14.87 \\
\hline Normalized NN & -10 & 15.19 & 3.96 & 5.37 \\
\hline & 10 & 29.21 & 17.32 & 17.62 \\
\hline & -6 & 19.42 & 9.91 & 8.23 \\
\hline & -3 & 21.88 & 12.78 & 11.43 \\
\hline & 0 & 25 & 16.48 & 14.59 \\
\hline & 3 & 27.9 & 19.39 & 18.03 \\
\hline & 6 & 29.76 & 21.62 & 20.82 \\
\hline & 10 & 33.35 & 26.42 & 23.43 \\
\hline
\end{tabular}


International Journal on Cybernetics \& Informatics (IJCI) Vol. 5, No. 4, August 2016

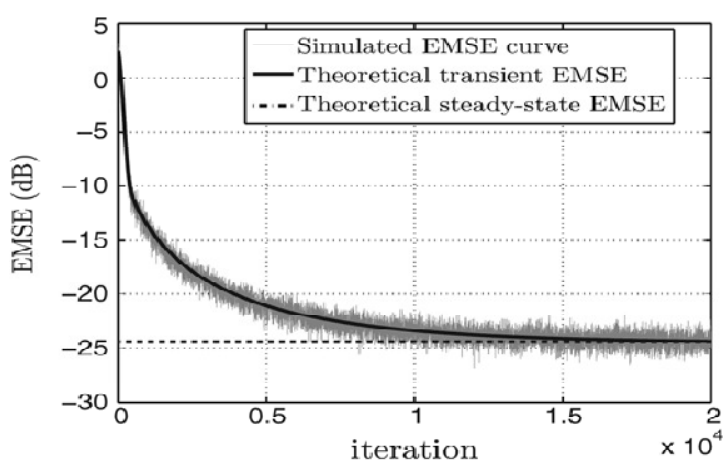

(a)

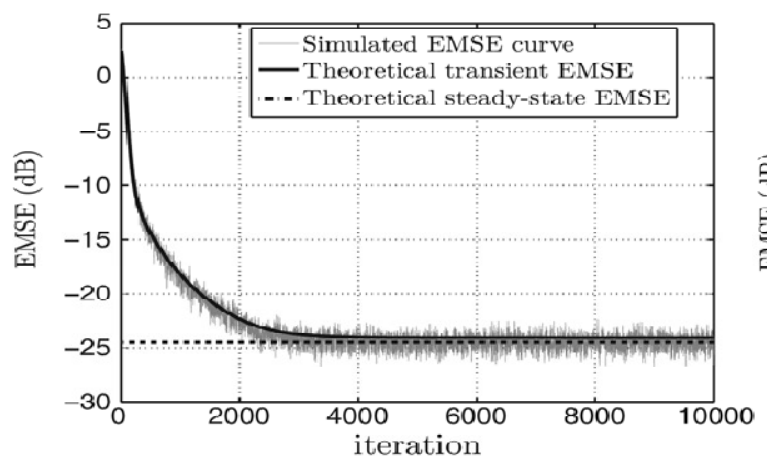

(c)

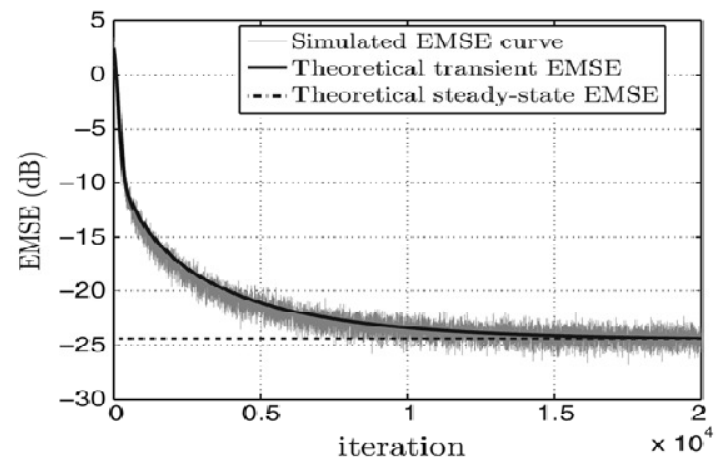

(b)

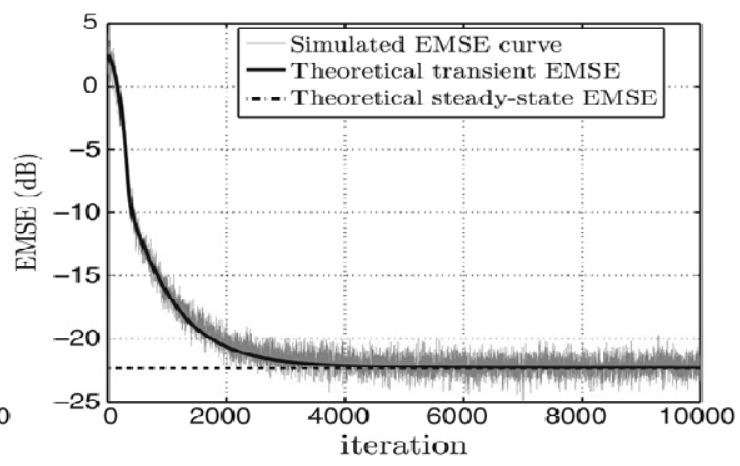

(d)

Figure 4: Different EMSE evaluation of NNLMS and its Types

\section{CONCLUSION}

In the proposed constructive approach in addition to the artifacts removal a performance comparisons made in the part of analysis of cardiac data under different noise environments. Also excesses Mean Square Error is observed on different cardiac data in steady state, transient using NNLMS and its variants. The quantitative analysis will provide the performance analysis of adaptive algorithms by weight updates under different filter taps environments. Finally the results are found to be satisfactory in few aspects like EMSE, filter taps and weight updating models.

\section{REFERENCES}

[1] "Nonnegative independent component analysis algorithms", by M. D. Plumbley in IEEE Trans. Neural Networks., Mar. 2003,Vol. 14, no. 3, PP. 534-543.

[2] "Separation of non-negative mixture of non-negative sources using a Bayesian approach and MCMC sampling," by A. Mohammad-Djafari, D. Brie, S. Moussaoui, and C. Carteret in IEEE Trans. Signal Processing., Nov. 2006, Vol. 54, no. 11, PP. 4133-4145.

[3] "Bayesian regularization and nonnegative de-convolution for room impulse response estimation," by D. D. Lee and Y. Lin, in IEEE Trans. Signal Processing., Mar. 2006 Vol. 54, no. 3, PP. 839-84.

[4] Adaptive Filter Theory, by Simon. Hay kin, 4th Edition: Pearson Education India, 2005 Delhi, India.

[5] "Fixed point error analysis of the normalized ladder algorithms," by V. U. Reddy and C. Samson in IEEE Trans. Acoustic., Speech, Signal Processing., Oct. 1983, Vol. 10, PP. 1177-1191. 
International Journal on Cybernetics \& Informatics (IJCI) Vol. 5, No. 4, August 2016

[6] "A statistical analysis of the affine projection algorithm for unity step size and autoregressive inputs," by J.-C. M. Bermudez, S. J. M. Almeida and N. J. Bershad, in IEEE Trans. Circuits Sysems. I: Fund. Theory Applications, Jul. 2005 Vol. 52, no. 7, PP. 1394-1405.

[7] "Geometric un mixing of large hyper spectral images: A barycentre coordinate approach," C. Richard and P. Honeine IEEE Trans. Geosciences and .Remote Sensing, 2012,Vol. 50, no. 6, PP. 2185-2195.

[8] "Nonnegative Matrix and Tensor Factorizations: Applications to Exploratory Multi-way Data Analysis and Blind Source Separation", R. Zdunek, A. Cichocki, and A. H. Phan, Hoboken, 2009 NJ, USA: Wiley,.

[9] "Nonnegative least-squares image de blurring: Improved gradient projection approaches," by R. Zanella, L. Zanni, F. Benvenuto and M. Bertero, in Inv. Probl., Feb. 2010, Vol. 26, no. 1, p. 025004.

[10] "Spectral unmixing," by J. F. Mustard and N. Keshava, in IEEE Signal Processing, Jan. 2002 Vol. 19, no. 1, PP. 44-57.

[11] "Realtime multiple pitch and multiple-instrument recognition for music signals using sparse nonnegative constraints," by S. Dubinov e.t.c, in Proc. 10th International . Conference Sep. 2007 Bordeaux, France, PP. 85-92.

\section{AUTHORS}

Dr. K. Chandra Bhushana Rao is working as Associate Professor, Dept. of ECE, JNTUK-University College of Engineering, VIZIANAGARAM. He received his B.E (ECE) degree from the Andhra University, Visakhapatnam, M.E (Communication Systems) from the Andhra University, Visakhapatnam, and Ph.D from Andhra University, Visakhapatnam. His area of interests is Microwave Communications and Signal Processing

Mrs. M. Madhavi is working as Associate Professor, Dept. of ECE, DVR \& Dr.HS MIC College of Technology, Kanchikacharla, Krishna (D). She received her B.Tech, from JNTU Hyderabad and M.Tech (DECS) from Jawaharlal Nehru Technological University, Hyderabad in 2008. Her areas of interests is Signal Processing and communications.
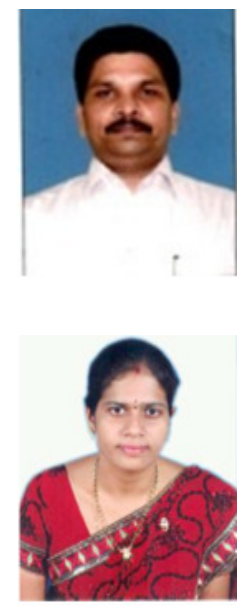